\title{
Green Chemistry Approach to Fast and Highly Efficient One-Pot Synthesis of Bis-Isoxazolyl-1,2,5,6-Tetrahydro Pyridine-3-Carboxylates
}

\author{
Eligeti Rajanarendar*, Kammari Thirupathaiah, Saini Rama Krishna, Baireddy Kishore \\ Department of Chemistry, Kakatiya University, Warangal, India \\ Email: " rajanarendareligeti@gmail.com
}

Received February 16, 2013; revised March 18, 2013; accepted March 26, 2013

Copyright $(0) 2013$ Eligeti Rajanarendar et al. This is an open access article distributed under the Creative Commons Attribution License, which permits unrestricted use, distribution, and reproduction in any medium, provided the original work is properly cited.

\begin{abstract}
The synthesis of the bis-isoxazolyl-1,2,5,6-tetrahydro pyridine-3-carboxylates is achieved in high yield without the production of toxic waste products by using room temperature ionic liquid (RTILs) triethyl ammonium acetate (TEAA). The RTIL TEAA played the dual role of efficient green solvent as well as recyclable catalyst.
\end{abstract}

Keywords: Bis-Isoxazolyl-1,2,5,6-Tetrahydro Pyridine-3-Carboxylates; Bis-Isoxazolyl-1,2,5,6-Tetrahydro Pyridine-3-Carboxylates; Green Synthesis

\section{Introduction}

Recently, there has been an increasing interest in the development of cleaner technologies to replace hazardous organic solvents with environmental benign solvents. Nowadays green chemistry emphasizes the optimization of synthetic methodologies to reduce pollution, cost and tedious work-ups. This new challenge has led to a growing interest in the field of organic synthesis. Among the several aspects of green chemistry, the reduction/replacement of volatile organic solvents from the reaction medium is of utmost importance. The use of a large excess of conventional volatile solvents required to conduct a chemical reaction creates ecological and economic concerns. The search for a nonvolatile and recyclable alternative solvent is thus holding a key role in this field of research. The use of fused organic salts, consisting of ions, is now emerging as a possible alternative. A proper choice of cations and anions is required to achieve ionic salts that are liquids at room temperature and are appropriately termed room temperature ionic liquids (RTILs). Common RTILs consists of $N, N^{\prime}$-dialkylimidazolium, alkylammonium, alkylphosphonium or $N$-alkylimidazolium as cations [1]. These innovative fluids are organic salts, whose cation, anion and the alkyl chain attached to organic cation can be varied to change their chemical and physical properties as per the needs of the chemical

${ }^{*}$ Corresponding author. process. A key feature of these liquids is their intrinsic ability to solvate a wide array of organic and inorganic substrates. They are blessed with qualities such as incredibly large liquidous range, negligible vapour pressure and recyclability [2]. In short, these ionic liquids have enlightened the way to environmentally benign procedures (3). The development of cleaner technologies is a major emphasis in green chemistry.

Several compounds consisting of reduced pyridine, like the 1,2,3,6-tetrahydropyridine $\mathbf{1}$ ring system is known to exhibit a variety of biological activities [4,5]. 1-Methyl-4-phenyl-1,2,3,6-tetrahydropyridine (MPTP) 2 was recently found to be neurotoxic, causing persistent parkinsonism in humans and other animal species [6-9]. Arecoline 3, an alkaloid containing tetrahydropyridine ring system obtained from the betel nut (Areca catechu), the fruit of a palm tree, has been used previously as a leading centrally active muscarinic agent [10]. 3-Methoxy-5-methyl-4,5,6,7-tetrahydroisoxazolo[4,5-c]-pyridinium 4 [11-13] and 3-methoxy-5-methyl-6,7-di-hydro4H-thiopyrano[3,4-d]isoxazol-5-ium 5 [14] salts are muscarinic agonists and partial agonists bio iso-sterically derived from arecoline (Figure 1). Biological activity of substituted isoxazoles has made them a focus of medicinal chemistry over the years. Spirocyclic isoxazoles such as aerothionin, aerophobin-1, and zamamistatin are biologically active alkaloids [15]. Isoxazoles are potent analgesic, anti-inflammatory [16], antimicrobial [17], COX-2 
<smiles>CN1CC=C(c2ccccc2)CC1</smiles>

1
2<smiles>COC(=O)C1=CCCN(C)C1</smiles>

3<smiles>COc1noc2c1C[NH+](C)CC2</smiles>

4<smiles>COc1noc2c1C[S+](C)CC2</smiles>

5

Figure 1. Biologically active tetrahydropyridines and arecoline analogs.

inhibitory [18], antitubercular [19], anticonvulsant [20], and anticancer agents [21]. In continuation of our studies on the synthesis of biologically important heterocyclic compounds [22-26], in the present work we have designed the synthesis of a novel tetrahydropyridine derivatives incorporated with isoxazole moiety utilizing an eco-friendly, reusable and inexpensive ionic liquid as solvent as well as a catalyst.

\section{Results and Discussion}

Literature search on the synthesis of tetrahydropyridines provide only few previous reports which utilizes the primary amine, aromatic aldehydes and $\beta$-diketones [27$34]$ for their synthesis. Therefore, we have further investigated this molecule of interest using greener approach and in search of better alternative over reported methods. Triethyl ammonium acetate (TEAA) ionic liquid (IL) has been found to be effective in aza/thia Michael reactions [35]. We report for the first time eco-friendly method for the synthesis of bis-isoxazolyl-1,2,5,6-tetrahydro pyridine-3-carboxylates by using cost effective TEAA ionic liquid (Scheme 1). To optimize the reaction condition, initial studies were conducted on the reaction of the isoxazole amine $\mathbf{1}$, aromatic aldehyde 2 and $\beta$-ketone ester 3 as a model reaction in the presence of different catalysts and solvents. The reaction afforded bis-isoxazolyl-1,2,5,6-tetrahydro pyridine-3-carboxy-lates $\mathbf{4}$ in 30\% - $75 \%$ yields at $60^{\circ} \mathrm{C}$ (Table 1 ).

We further explored the reaction in the presence of TEAA ionic liquid. We started the five component reaction by examining the effect of TEAA ionic liquid on yield and time of the typical reaction of isoxazole amine $\mathbf{1}$, aromatic aldehyde $\mathbf{2}$ and $\beta$-ketone ester $\mathbf{3}$ at room temperature. To our surprise, the use of TEAA ionic liquid decreased reaction time, temperature and resulted in improved yields. We also carried out the reaction in the presence of different ILs at room temperature. The results are summarized in Table 2. Among the ILs examined TEAA proved to be the most effective in terms of yields, reaction time, and more recyclability. Utilization of other ILs was found to be quite unsatisfactory.

Having established that, the best solvent and catalyst is TEAA for this transformation, the another advantage is,

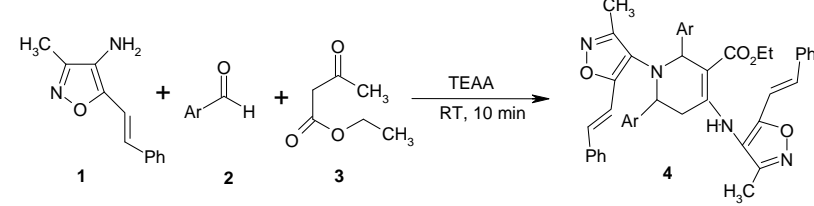

Scheme 1. Synthesis of bis-isoxazolyl-1,2,5,6-tetrahydro pyridine-3-carboxylates by using TEAA IL.

Table 1. Effect of various catalysts and solvents on the synthesis of bis-isoxazolyl-1,2,5,6-tetrahydro pyridine-3-carboxylates.
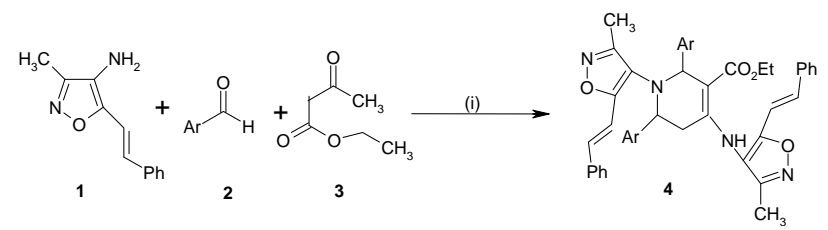

\begin{tabular}{ccccc}
\hline Entry & Catalyst $(10$ mol\%) & Solvent & Time (min) & Yield $^{\mathrm{b}}(\%)$ \\
\hline 1 & $\mathrm{CAN}$ & $\mathrm{THF}$ & 30 & 75 \\
2 & Silica gel & $\mathrm{EtOH}$ & 50 & 60 \\
3 & $\mathrm{I}_{2}$ & $\mathrm{CH}_{3} \mathrm{CN}$ & 46 & 50 \\
4 & $\mathrm{InCl}_{3}$ & $\mathrm{CH}_{3} \mathrm{CN}$ & 80 & 42 \\
5 & $\mathrm{~L}$ proline $+\mathrm{TFA}$ & $\mathrm{CH}_{3} \mathrm{CN}$ & 74 & 45 \\
6 & $p \mathrm{TSA}_{7}$ & $\mathrm{DMSO}$ & 55 & 48 \\
7 & $\mathrm{ZnCl}_{2}$ & $\mathrm{EtOH}$ & 45 & 30 \\
\hline
\end{tabular}

${ }^{\mathrm{a}}$ Reaction conditions: (i) isoxazole amine $\mathbf{1}(2 \mathrm{mmol})$, aldehyde $2(2 \mathrm{mmol})$, EAA $3(1 \mathrm{mmol})$ and catalyst $10 \mathrm{~mol} \%$ in solvent $(10 \mathrm{~mL}), 60^{\circ} \mathrm{C}^{\mathrm{b}}$. Isolated yields.

Table 2. Optimization of reaction condition by using different ILs. ${ }^{\mathrm{a}}$

\begin{tabular}{cccc}
\hline Entry & IL & Time (min) & Yield $^{\mathrm{b}}(\%)$ \\
\hline 1 & TEAA & 10 & 98 \\
2 & {$[\mathrm{bmIm}] \mathrm{BF}_{4}$} & 20 & 45 \\
3 & {$[\mathrm{bmIm}] \mathrm{OH}$} & 45 & 20 \\
4 & {$[\mathrm{bmIm}] \mathrm{Br}$} & 35 & 15 \\
5 & {$\left[\mathrm{HMIm}_{\mathrm{B} F}\right.$} & 60 & 65 \\
\hline
\end{tabular}

${ }^{\mathrm{a}}$ Reaction conditions: isoxazole amine $1(2 \mathrm{mmol})$, aldehyde $2(2 \mathrm{mmol})$, EAA 3 (1 mmol) and IL (5 mL), RT. ' Isolated yields. 
it can be easily recovered after the completion of the reaction and can be reused in subsequent runs (five runs) without loss of efficiency and with negligible loss of IL (Table 3 ). The products were easily separated by simple extraction with ether and characterized by IR, ${ }^{1} \mathrm{H}$ NMR, ${ }^{13} \mathrm{C}$ NMR and mass spectroscopy and also by analytical data.

To investigate the scope of TEAA IL under the optimized reaction conditions, a number of bis-isoxazolyl-1, 2,5,6-tetrahydro pyridine-3-carboxylate derivatives were synthesized and the results are summarized in Table 4. In all the cases, the products were obtained in excellent yields. The great advantages of the present procedure using TEAA in the synthesis of bis-isoxazolyl-1,2,5,6tetrahydro pyridine-3-carboxylates are the mild conditions, high yields, short reaction times, simple experimental set-up and workup. Besides this, it is equally efficient and is compatible with different functional groups (electron releasing and electron attracting groups) and the approach proved to be of general applicability.

The plausible mechanism for the synthesis of bisisoxazolyl-1,2,5,6-tetrahydro pyridine-3-carboxylates involves the formation of imine (5) and enamine (6) initially in the presence of IL. Then the enamine makes a nucleophilic attack on to imine, to form a Mannich type intermediate (7). This Mannich type intermediate (7) reacts with aldehyde to form (8) with the elimination of water molecule with the influence of IL. 8 undergoes spontaneous tautomerization and converts into (9). The tautomer (9) undergoes intramolecular Mannich type reaction to form intermediate (10), which tautomerises spontaneously to form bis-isoxazolyl-1,2,5,6-tetrahydro pyridine-3-carboxylate (4) (Scheme 2).

\section{Conclusions}

In conclusion, the present procedure that uses ammonium ionic liquid TEAA provides a fast and highly efficient methodology for the synthesis of bis-isoxazolyl-1,2, 5,6-tetrahydro pyridine-3-carboxylates. The significant improvements noticed in this reaction are high yields, reduction in reaction time and avoids the usage of hazardous organic solvents. These reactions were clean, and

Table 3. Recycling of IL TEAA.

\begin{tabular}{ccc}
\hline Run & IL recycled (in $\mathrm{mL})$ & Yield $^{\mathrm{a}}(\%)$ \\
\hline 1 & 5 & 97 \\
2 & 5 & 97 \\
3 & 4.8 & 95 \\
4 & 4.6 & 94 \\
5 & 4.2 & 93 \\
\hline
\end{tabular}

${ }^{\mathrm{a}}$ Isolated yields. no by-products were detected under these conditions. To the best of our knowledge, this happens to be the first report for the construction of tetrahydropyridine ring by using isoxazole amine, aldehyde and EAA with TEAA ionic liquid, in which ionic liquid playing the dual role of solvent as well as catalyst. Moreover, this work clearly demonstrates that the potential of room temperature ionic liquid TEAA, to act as an efficient and recyclable catalyst, green solvent and shows much promise for future applications.

The authors are thankful to the Head, Department of Chemistry, Kakatiya University, Warangal for providing the facilities, the Director, Indian Institute of Chemical Technology, Hyderabad for recording ${ }^{1} \mathrm{H}$ NMR, and Mass Spectral data. B. Kishore is thankful to UGC, New Delhi, for the award of a fellowship (JRF).

\section{Experimental Part}

All the melting points were determined on a Fisher-Johns melting point apparatus and are uncorrected. Analytical TLC was performed on Merck precoated $60 \mathrm{~F}_{254}$ silica gel plates. Visualization was done by exposure to iodine vapor. IR spectra ( $\mathrm{KBr}$ pellet) were recorded on a Perkin Elmer BX series FT-IR spectrometer. ${ }^{1} \mathrm{H}$ NMR spectra were recorded on a Varian Gemini $300 \mathrm{MHz}$ spectrometer. ${ }^{13} \mathrm{C}$ NMR spectra were recorded on a Bruker $75 \mathrm{MHz}$ spectrometer. Chemical shift values are given in ppm $(\delta)$ with tetramethyl silane as an internal standard. Mass spectral measurements were carried out by EI method on a Jeol JMC-300 spectrometer at $70 \mathrm{eV}$. Elemental analyses were performed on a Carlo Erba 106 and Perkin Elmer model 240 analyzers.

The IL TEAA was prepared according to the reported procedure [36-38].

\subsection{Green Chemistry Approach of Fast and Highly Efficient One-Pot Synthesis of Bisisoxazolyl-1,2,5,6-Tetrahydro Pyridine-3-Carboxylates; Typical Procedure}

4-Amino-3-methyl-5-styrylisoxazole 1 (1 mmol), benzaldehyde 2a (1 mmol), and ethyl aceto acetate 3 (0.5 mmol) were taken in IL TEAA $(5 \mathrm{~mL})$, and then reaction mixture was stirred at room temperature for $10 \mathrm{~min}$. After the completion of the reaction (monitored by TLC), the reaction mixture was extracted with diethyl ether. The combined layers was separated and dried over anhyd. $\mathrm{Na}_{2} \mathrm{SO}_{4}$, and evaporated under reduced pressure to afford the crude product 4a. The ionic liquid left over in the reaction was washed with ethyl acetate and dried at $80^{\circ} \mathrm{C}$ under vacuum and was reused for 5 consecutive runs. This procedure was followed for all the reactions listed in the Table 4. 

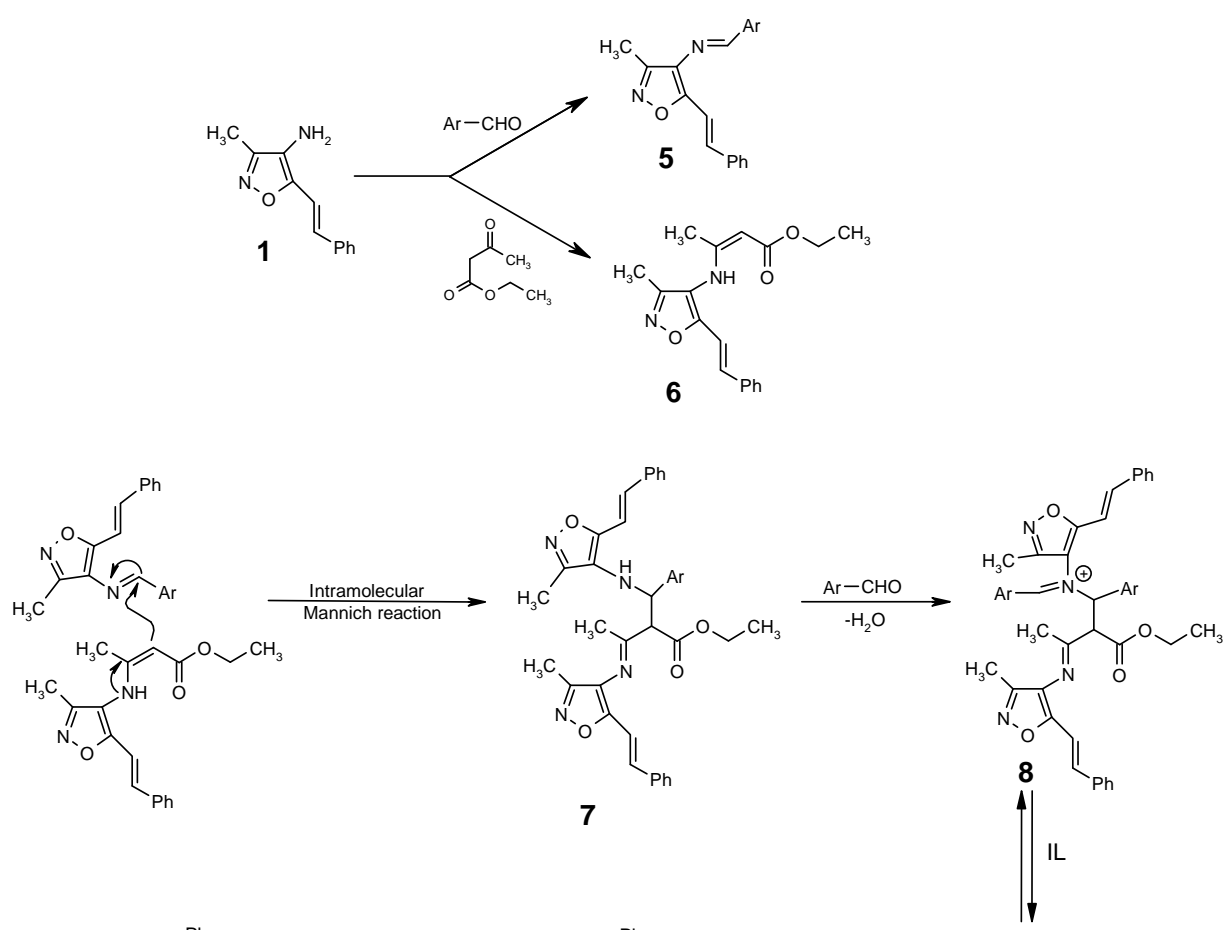

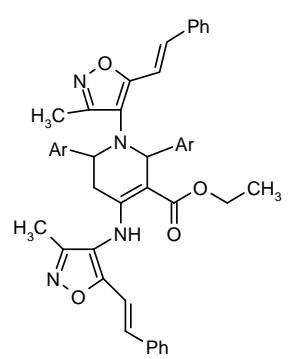

4

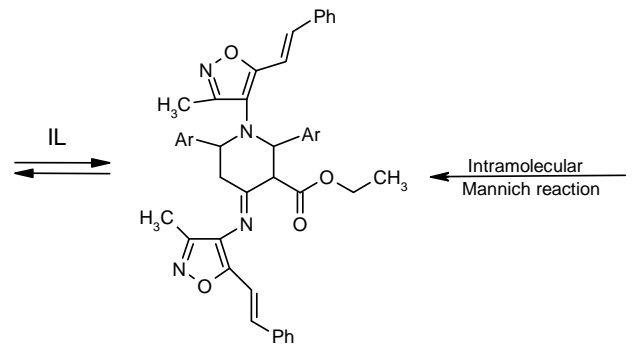

10

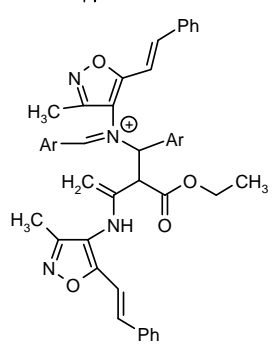

9

Scheme 2. Plausible mechanism for the formation of bis-isoxazolyl-1,2,5,6-tetrahydro pyridine-3-carboxylates.

Table 4. Synthesis of bis-isoxazolyl-1,2,5,6-tetrahydro pyridine-3-carboxylates by using TEAA IL. ${ }^{\text {a }}$

Entry Isoxazole amine 1




\section{Continued}

3<smiles>Cc1noc(/C=C/c2ccccc2)c1N</smiles>

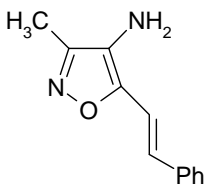<smiles>Cc1noc(/C=C/c2ccccc2)c1N</smiles><smiles>Cc1noc(/C=C/c2ccccc2)c1N</smiles>

1
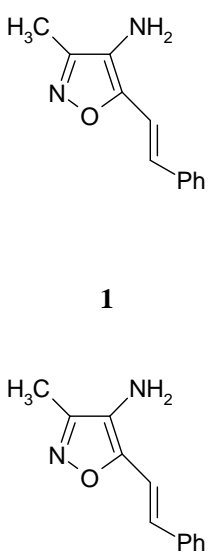<smiles>Cc1ccc(C=O)cc1</smiles>

2c<smiles>O=Cc1ccccc1Cl</smiles><smiles>O=Cc1ccc(Cl)cc1Cl</smiles>

$2 \mathbf{e}$<smiles>O=Cc1ccccc1Br</smiles>

$2 f$<smiles>O=Cc1ccc(Br)cc1Br</smiles>

$2 \mathrm{~g}$<smiles>O=Cc1ccccc1O</smiles>

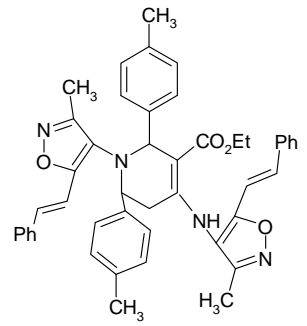

4c
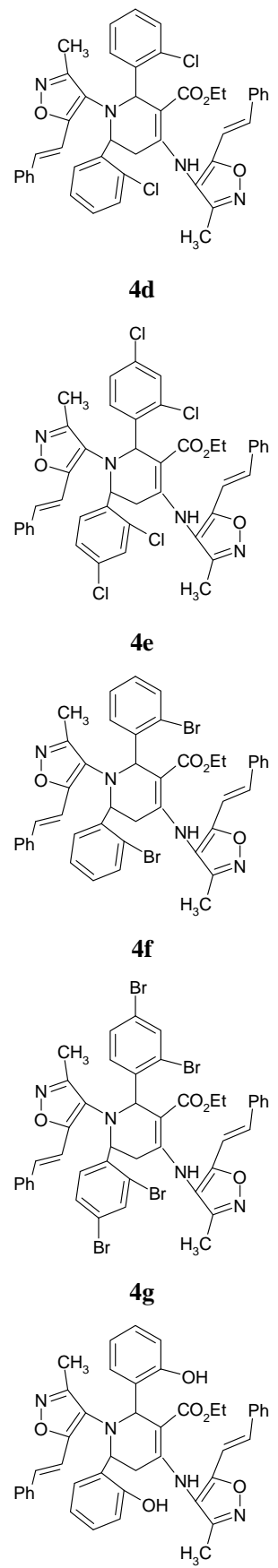

4d

$4 e$

$4 f$

10 4g (97)

4d (96)

9 4e (94) 4f (94)

4h 


\section{Continued}

9<smiles>Cc1noc(/C=C/c2ccccc2)c1N</smiles><smiles>Cc1noc(/C=C/c2ccccc2)c1N</smiles>

1

11<smiles>Cc1noc(/C=C/c2ccccc2)c1N</smiles><smiles>Cc1noc(/C=C/c2ccccc2)c1N</smiles>

1<smiles>Cc1noc(/C=C/c2ccccc2)c1N</smiles>

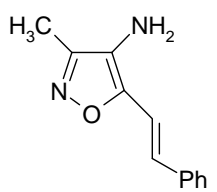

14<smiles>O=Cc1ccccc1[N+](=O)[O-]</smiles><smiles>O=Cc1ccc([N+](=O)[O-])cc1</smiles>

$2 \mathbf{j}$<smiles>CN(C)c1ccc(C=O)cc1</smiles>

$2 \mathbf{k}$<smiles>O=Cc1cccs1</smiles>

21<smiles>O=Cc1ccc[nH]1</smiles>

$2 m$<smiles>O=Cc1ccco1</smiles>

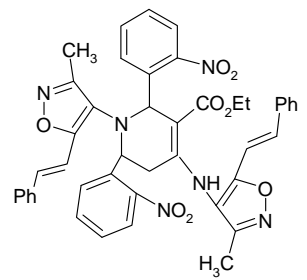

$4 i$

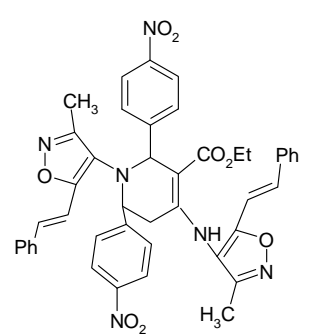

$4 j$

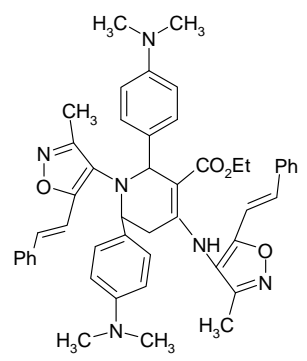

$4 k$

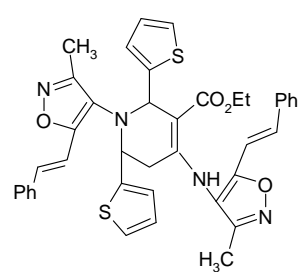

41

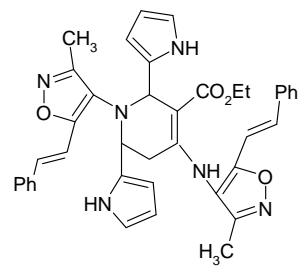

$4 m$

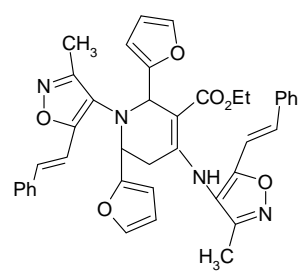

4i (96)

$4 n$

${ }^{a}$ Reaction conditions: isoxazole amine 1 ( $\left.2 \mathrm{mmol}\right)$, aldehyde 2 ( $\left.2 \mathrm{mmol}\right)$, EAA 3 (1 mmol) and TEAA IL $(5 \mathrm{~mL})$, RT stirring. ${ }^{\mathrm{b}}$ Isolated yields. 


\subsection{Spectral Data of Compounds (4a-n)}

Ethyl-4-(3-methyl-5-styrylisoxazol-4-ylamino)-1,2,5,6-tet rahydro-1-(3-methyl-5-styrylisoxazol-4-yl)-2,6-diphenyl pyridine-3-carboxylate (4a)

Brown solid; $\mathrm{mp} 221^{\circ} \mathrm{C}-223^{\circ} \mathrm{C}$. IR (KBr): 1665,3428 $\mathrm{cm}^{-1} .{ }^{1} \mathrm{H}$ NMR $\left(300 \mathrm{MHz}, \mathrm{CDCl}_{3}\right): \delta=1.32(\mathrm{t}, 3 \mathrm{H}, \mathrm{J}=$ $\left.7.2 \mathrm{~Hz}, \mathrm{OCH}_{2} \mathrm{CH}_{3}\right), 2.23$ (s, $6 \mathrm{H}, 2$ isoxazole- $\left.\mathrm{CH}_{3}\right), 2.62$ $(\mathrm{dd}, 1 \mathrm{H}, \mathrm{CH}, \mathrm{J}=15.2,2.4 \mathrm{~Hz}), 2.76(\mathrm{dd}, 1 \mathrm{H}, \mathrm{CH}, \mathrm{J}=$ $15.2,5.7 \mathrm{~Hz}), 4.10\left(\mathrm{q}, 2 \mathrm{H}, \mathrm{OCH}_{2} \mathrm{CH}_{3}\right), 5.23(\mathrm{~s}, 1 \mathrm{H}$, Ar-CH), 6.09 (t, $1 \mathrm{H}, \mathrm{Ar}-\mathrm{CH}), 6.68(\mathrm{~d}, 1 \mathrm{H}, \mathrm{CH}=\mathrm{CH}, \mathrm{J}=$ $12 \mathrm{~Hz}), 6.83(\mathrm{~d}, 1 \mathrm{H}, \mathrm{CH}=\mathrm{CH}, \mathrm{J}=12 \mathrm{~Hz}), 6.98-7.91$ (m, $20 \mathrm{H}, \mathrm{ArH}), 8.93\left(\mathrm{~s}, 1 \mathrm{H}, \mathrm{NH}, \mathrm{D}_{2} \mathrm{O}\right.$ exchangeable). ${ }^{13} \mathrm{C}$ NMR $\left(75 \mathrm{MHz}, \mathrm{DMSO}-\mathrm{d}_{6}\right): \delta=10.31,12.36,32.42$, $48.46,54.16,63.45,101.42,104.26,123.42,126.13$, $127.32,128.06,128.68,129.21,134.61,136.52,139.11$, 141.46, 151.34, 158.56. MS (ESI): $m / z=689.36[\mathrm{M}+\mathrm{H}]^{+}$. Anal. Calcd for $\mathrm{C}_{44} \mathrm{H}_{40} \mathrm{~N}_{4} \mathrm{O}_{4}$ : C, 76.72; H, 5.85; N, 8.13. Found. C, 76.68; H, 5.81; N, 8.19.

Ethyl 4-(3-methyl-5-styrylisoxazol-4-ylamino)-1,2,5, 6-tetrahdro-2,6-bis(4-methoxyphenyl)-1-(3-methyl-5-styr ylisoxazol-4-yl)pyridine-3-carboxylate (4b)

Yellow solid; $\mathrm{mp} 215^{\circ} \mathrm{C}-217^{\circ} \mathrm{C}$. IR (KBr): 1661,3442 $\mathrm{cm}^{-1} .{ }^{1} \mathrm{H}$ NMR $\left(300 \mathrm{MHz}, \mathrm{CDCl}_{3}\right): \delta=1.26(\mathrm{t}, 3 \mathrm{H}, \mathrm{J}=$ $\left.7.2 \mathrm{~Hz}, \mathrm{OCH}_{2} \mathrm{CH}_{3}\right), 2.30$ (s, $6 \mathrm{H}, 2$ isoxazole- $\left.\mathrm{CH}_{3}\right), 2.59$ $(\mathrm{dd}, 1 \mathrm{H}, \mathrm{CH}, \mathrm{J}=15.2,2.4 \mathrm{~Hz}), 2.74(\mathrm{dd}, 1 \mathrm{H}, \mathrm{CH}, \mathrm{J}=$ $15.2,5.7 \mathrm{~Hz}), 3.62\left(\mathrm{~s}, 6 \mathrm{H}, 2 \mathrm{OCH}_{3}\right), 4.08(\mathrm{q}, 2 \mathrm{H}$, $\left.\mathrm{OCH}_{2} \mathrm{CH}_{3}\right), 5.24(\mathrm{~s}, 1 \mathrm{H}, \mathrm{Ar}-\mathrm{CH}), 6.04(\mathrm{t}, 1 \mathrm{H}, \mathrm{Ar}-\mathrm{CH})$, $6.68(\mathrm{~d}, 1 \mathrm{H}, \mathrm{CH}=\mathrm{CH}, \mathrm{J}=12 \mathrm{~Hz}), 6.72(\mathrm{~d}, 1 \mathrm{H}, \mathrm{CH}=$ $\mathrm{CH}, \mathrm{J}=12 \mathrm{~Hz}$ ), $7.00-7.96$ (m, $18 \mathrm{H}, \mathrm{ArH}), 9.00$ (s, $1 \mathrm{H}$, $\mathrm{NH}, \mathrm{D}_{2} \mathrm{O}$ exchangeable). ${ }^{13} \mathrm{C}$ NMR (75 MHz, DMSO-d 6 ): $\delta=10.26,12.41,32.40,48.42,54.28,63.37,63.81$, $101.36,104.55,123.32,126.41,128.21,128.57,129.17$, 134.48, 136.44, 139.23, 141.38, 151.40, 158.32, 159.13 . MS (ESI): $m / z=749.37[\mathrm{M}+\mathrm{H}]^{+}$. Anal. Calcd for $\mathrm{C}_{46} \mathrm{H}_{44} \mathrm{~N}_{4} \mathrm{O}_{6}: \mathrm{C}, 73.78 ; \mathrm{H}, 5.92 ; \mathrm{N}, 7.48$. Found. C, 73.82; $\mathrm{H}, 5.87 ; \mathrm{N}, 7.41$.

Ethyl 4-(3-methyl-5-styrylisoxazol-4-ylamino)-1,2,5, 6-tetrahydro-1-(3-methyl-5-styrylisoxazol-4-yl)-2,6-diptolylpyridine-3-carboxylate (4c)

Brown solid; $\mathrm{mp} 211^{\circ} \mathrm{C}-213^{\circ} \mathrm{C}$. IR (KBr): 1661, 3442 $\mathrm{cm}^{-1} .{ }^{1} \mathrm{H}$ NMR $\left(300 \mathrm{MHz}, \mathrm{CDCl}_{3}\right): \delta=1.09(\mathrm{t}, 3 \mathrm{H}, \mathrm{J}=$ $\left.7.2 \mathrm{~Hz}, \mathrm{OCH}_{2} \mathrm{CH}_{3}\right), 2.25$ (s, 6H, 2isoxazole- $\left.\mathrm{CH}_{3}\right), 2.46$ (s, $\left.6 \mathrm{H}, 2 \mathrm{Ar}-\mathrm{CH}_{3}\right), 2.50(\mathrm{dd}, 1 \mathrm{H}, \mathrm{CH}, \mathrm{J}=15.2,2.4 \mathrm{~Hz}$ ), $2.71(\mathrm{dd}, 1 \mathrm{H}, \mathrm{CH}, \mathrm{J}=15.2,5.7 \mathrm{~Hz}), 4.11(\mathrm{q}, 2 \mathrm{H}$, $\left.\mathrm{OCH}_{2} \mathrm{CH}_{3}\right), 5.24$ (s, $\left.1 \mathrm{H}, \mathrm{Ar}-\mathrm{CH}\right), 6.00(\mathrm{t}, 1 \mathrm{H}, \mathrm{Ar}-\mathrm{CH})$, $6.61(\mathrm{~d}, 1 \mathrm{H}, \mathrm{CH}=\mathrm{CH}, \mathrm{J}=12 \mathrm{~Hz}), 6.74(\mathrm{~d}, 1 \mathrm{H}, \mathrm{CH}=$ $\mathrm{CH}, \mathrm{J}=12 \mathrm{~Hz}$ ), $7.11-7.83(\mathrm{~m}, 18 \mathrm{H}, \mathrm{ArH}), 8.90(\mathrm{~s}, 1 \mathrm{H}$, $\mathrm{NH}, \mathrm{D}_{2} \mathrm{O}$ exchangeable). ${ }^{13} \mathrm{C}$ NMR (75 MHz, DMSO-d $\mathrm{d}_{6}$ ): $\delta=10.21,12.33,24.23,32.26,48.39,54.22,63.40$, $101.56,104.31,123.40,126.23,128.18,128.52,129.19$, 134.68, 136.50, 138.37, 139.05, 141.42, 151.39, 158.44. MS (ESI): $m / z=717.17[\mathrm{M}+\mathrm{H}]^{+}$. Anal. Calcd for $\mathrm{C}_{46} \mathrm{H}_{44} \mathrm{~N}_{4} \mathrm{O}_{4}$ : C, 77.07; H, 6.19; N, 7.82. Found. C, 77.12;
$\mathrm{H}, 6.16 ; \mathrm{N}, 7.80$.

Ethyl 4-(3-methyl-5-styrylisoxazol-4-ylamino)-2, 6-bis (2-chlorophenyl)-1,2,5,6-tetrahydro-1-(3-methyl-5-styrylisoxazol-4-yl)pyridine-3-carboxylate (4d)

Pale yellow solid; $\mathrm{mp} 235^{\circ} \mathrm{C}-237^{\circ} \mathrm{C}$. IR (KBr): 1668 , $3440 \mathrm{~cm}^{-1} .{ }^{1} \mathrm{H}$ NMR $\left(300 \mathrm{MHz}, \mathrm{CDCl}_{3}\right): \delta=1.12(\mathrm{t}, 3 \mathrm{H}$, $\left.\mathrm{J}=7.2 \mathrm{~Hz}, \mathrm{OCH}_{2} \mathrm{CH}_{3}\right), 2.22\left(\mathrm{~s}, 6 \mathrm{H}, 2\right.$ isoxazole- $\left.\mathrm{CH}_{3}\right)$, $2.53(\mathrm{dd}, 1 \mathrm{H}, \mathrm{CH}, \mathrm{J}=15.2,2.4 \mathrm{~Hz}), 2.69(\mathrm{dd}, 1 \mathrm{H}, \mathrm{CH}, \mathrm{J}$ $=15.2,5.7 \mathrm{~Hz}), 4.07\left(\mathrm{q}, 2 \mathrm{H}, \mathrm{OCH}_{2} \mathrm{CH}_{3}\right), 5.20(\mathrm{~s}, 1 \mathrm{H}$, Ar-CH), 5.98 (t, $1 \mathrm{H}, \mathrm{Ar}-\mathrm{CH}), 6.64(\mathrm{~d}, 1 \mathrm{H}, \mathrm{CH}=\mathrm{CH}, \mathrm{J}=$ $12 \mathrm{~Hz}), 6.70(\mathrm{~d}, 1 \mathrm{H}, \mathrm{CH}=\mathrm{CH}, \mathrm{J}=12 \mathrm{~Hz}), 7.01-7.91$ $(\mathrm{m}, 18 \mathrm{H}, \mathrm{ArH}), 8.83\left(\mathrm{~s}, 1 \mathrm{H}, \mathrm{NH}, \mathrm{D}_{2} \mathrm{O}\right.$ exchangeable).${ }^{13} \mathrm{C}$ NMR (75MHz, DMSO-d 6 ): $\delta=10.24,12.33$, $32.41,48.46,54.09,63.31,101.40,104.19,123.39$, $126.24,127.39,128.61,129.33,134.21,134.59,136.47$, 139.01, 141.39, 151.30, 158.62. MS (ESI): $m / z=757.29$ $[\mathrm{M}+\mathrm{H}]^{+}$. Anal. Calcd for $\mathrm{C}_{44} \mathrm{H}_{38} \mathrm{Cl}_{2} \mathrm{~N}_{4} \mathrm{O}_{4}: \mathrm{C}, 69.75 ; \mathrm{H}$, $5.05 ; \mathrm{N}, 7.39$. Found. C, 69.81; H, 5.07; N, 7.33.

Ethyl 4-(3-methyl-5-styrylisoxazol-4-ylamino)-2,6-bis (2,4-dichlorophenyl)-1,2,5,6-tetrahydro-1-(3-methyl-5styrylisoxazol-4-yl)pyridine-3-carboxylate (4e)

Pale yellow solid; $\mathrm{mp} 228^{\circ} \mathrm{C}-230^{\circ} \mathrm{C}$. IR (KBr): 1665 , $3438 \mathrm{~cm}^{-1}$. ${ }^{1} \mathrm{H}$ NMR $\left(300 \mathrm{MHz}, \mathrm{CDCl}_{3}\right): \delta=1.10(\mathrm{t}, 3 \mathrm{H}$, $\left.\mathrm{J}=7.2 \mathrm{~Hz}, \mathrm{OCH}_{2} \mathrm{CH}_{3}\right), 2.20\left(\mathrm{~s}, 6 \mathrm{H}, 2\right.$ isoxazole- $\left.\mathrm{CH}_{3}\right)$, $2.49(\mathrm{dd}, 1 \mathrm{H}, \mathrm{CH}, \mathrm{J}=15.2,2.4 \mathrm{~Hz}), 2.60(\mathrm{dd}, 1 \mathrm{H}, \mathrm{CH}, \mathrm{J}$ $=15.2,5.7 \mathrm{~Hz}), 4.12\left(\mathrm{q}, 2 \mathrm{H}, \mathrm{OCH}_{2} \mathrm{CH}_{3}\right), 5.11(\mathrm{~s}, 1 \mathrm{H}$, Ar-CH), $5.86(\mathrm{t}, 1 \mathrm{H}, \mathrm{Ar}-\mathrm{CH}), 6.60(\mathrm{~d}, 1 \mathrm{H}, \mathrm{CH}=\mathrm{CH}, \mathrm{J}=$ $12 \mathrm{~Hz}), 6.73(\mathrm{~d}, 1 \mathrm{H}, \mathrm{CH}=\mathrm{CH}, \mathrm{J}=12 \mathrm{~Hz}), 6.98-7.87(\mathrm{~m}$, $16 \mathrm{H}, \mathrm{ArH}), 9.10$ (s, $1 \mathrm{H}, \mathrm{NH}, \mathrm{D}_{2} \mathrm{O}$ exchangeable). ${ }^{13} \mathrm{C}$ NMR $\left(75 \mathrm{MHz}\right.$, DMSO-d $\left.\mathrm{d}_{6}\right): \delta=11.09,12.41,32.38$, $48.67,54.03,63.29,101.38,104.20,123.57,126.26$, $128.55,129.36,134.38,134.56,134.78,136.42,139.35$, 141.51, 151.29, 158.58. MS (ESI): $m / z=825.40[\mathrm{M}+\mathrm{H}]^{+}$. Anal. Calcd for $\mathrm{C}_{44} \mathrm{H}_{36} \mathrm{Cl}_{4} \mathrm{~N}_{4} \mathrm{O}_{4}: \mathrm{C}, 63.93 ; \mathrm{H}, 4.39 ; \mathrm{N}$, 6.78. Found. C, 63.97; H, 4.33; N, 6.74.

Ethyl 4-(3-methyl-5-styrylisoxazol-4-ylamino)-2,6-bis (2-bromophenyl)-1,2,5,6-tetrahydro-1-(3-methyl-5-styryli soxazol-4-yl)pyridine-3-carboxylate (4f)

Pale brown solid; $\mathrm{mp} 224^{\circ} \mathrm{C}-226^{\circ} \mathrm{C}$. IR (KBr): 1670 , $3441 \mathrm{~cm}^{-1} .{ }^{1} \mathrm{H}$ NMR $\left(300 \mathrm{MHz}, \mathrm{CDCl}_{3}\right): \delta=1.09(\mathrm{t}, 3 \mathrm{H}$, $\left.\mathrm{J}=7.2 \mathrm{~Hz}, \mathrm{OCH}_{2} \mathrm{CH}_{3}\right), 2.28\left(\mathrm{~s}, 6 \mathrm{H}, 2\right.$ isoxazole- $\left.\mathrm{CH}_{3}\right)$, $2.41(\mathrm{dd}, 1 \mathrm{H}, \mathrm{CH}, \mathrm{J}=15.2,2.4 \mathrm{~Hz}), 2.63(\mathrm{dd}, 1 \mathrm{H}, \mathrm{CH}, \mathrm{J}$ $=15.2,5.7 \mathrm{~Hz}), 4.10\left(\mathrm{q}, 2 \mathrm{H}, \mathrm{OCH}_{2} \mathrm{CH}_{3}\right), 5.25(\mathrm{~s}, 1 \mathrm{H}$, Ar-CH), $5.78(\mathrm{t}, 1 \mathrm{H}, \mathrm{Ar}-\mathrm{CH}), 6.61(\mathrm{~d}, 1 \mathrm{H}, \mathrm{CH}=\mathrm{CH}, \mathrm{J}=$ $12 \mathrm{~Hz}), 6.69(\mathrm{~d}, 1 \mathrm{H}, \mathrm{CH}=\mathrm{CH}, \mathrm{J}=12 \mathrm{~Hz}), 7.05-7.93$ $(\mathrm{m}, 18 \mathrm{H}, \mathrm{ArH}), 9.08\left(\mathrm{~s}, 1 \mathrm{H}, \mathrm{NH}, \mathrm{D}_{2} \mathrm{O}\right.$ exchangeable). ${ }^{13} \mathrm{C}$ NMR (75 MHz, DMSO-d 6 ): $\delta=10.16,12.27$, $32.53,48.42,54.17,63.42,101.33,104.24,123.48$, $126.15,127.46,128.58,129.45,134.32,134.68,136.51$, 139.12, 141.26, 151.44, 158.78. MS (ESI): $\mathrm{m} / z=845.11$ $[\mathrm{M}+\mathrm{H}]^{+}$. Anal. Calcd for $\mathrm{C}_{44} \mathrm{H}_{38} \mathrm{Br}_{2} \mathrm{~N}_{4} \mathrm{O}_{4}: \mathrm{C}, 62.42 ; \mathrm{H}$, 4.52; N, 6.62. Found. C, 62.38; H, 4.51; N, 6.65.

Ethyl 4-(3-methyl-5-styrylisoxazol-4-ylamino)-2,6-bis (2,4-dibromophenyl)-1,2,5,6-tetrahydro-1-(3-methyl-5- 
styrylisoxazol-4-yl)pyridine-3-carboxylate (4g)

Brown solid; $\mathrm{mp} 241^{\circ} \mathrm{C}-243^{\circ} \mathrm{C}$. IR (KBr): 1668,3442 $\mathrm{cm}^{-1} .{ }^{1} \mathrm{H}$ NMR $\left(300 \mathrm{MHz}, \mathrm{CDCl}_{3}\right): \delta=1.12(\mathrm{t}, 3 \mathrm{H}, \mathrm{J}=$ $\left.7.2 \mathrm{~Hz}, \mathrm{OCH}_{2} \mathrm{CH}_{3}\right), 2.24\left(\mathrm{~s}, 6 \mathrm{H}, 2\right.$ isoxazole- $\left.\mathrm{CH}_{3}\right), 2.48$ (dd, $1 \mathrm{H}, \mathrm{CH}, \mathrm{J}=15.2,2.4 \mathrm{~Hz}), 2.59$ (dd, $1 \mathrm{H}, \mathrm{CH}, \mathrm{J}=$ $15.2,5.7 \mathrm{~Hz}), 4.20$ (q, $\left.2 \mathrm{H}, \mathrm{OCH}_{2} \mathrm{CH}_{3}\right), 5.19$ (s, $1 \mathrm{H}$, Ar-CH), 5.64 (t, 1 H, Ar-CH), 6.57 (d, $1 \mathrm{H}, \mathrm{CH}=\mathrm{CH}, \mathrm{J}=$ $12 \mathrm{~Hz}), 6.70(\mathrm{~d}, 1 \mathrm{H}, \mathrm{CH}=\mathrm{CH}, \mathrm{J}=12 \mathrm{~Hz}), 7.00-7.87$ (m, $16 \mathrm{H}, \mathrm{ArH}), 8.87$ (s, $1 \mathrm{H}, \mathrm{NH}, \mathrm{D}_{2} \mathrm{O}$ exchangeable). ${ }^{13} \mathrm{C}$ NMR (75 MHz, DMSO-d ${ }_{6}$ ): $\delta=10.14,12.27,24.38$, $32.39,48.25,54.20,63.43,101.51,104.44,123.57$, $126.34,128.11,128.39,129.10,134.56,136.41,138.28$, 139.22, 141.35, 151.46, 158.61. MS (ESI): $m / z=1001.08$ $[\mathrm{M}+\mathrm{H}]^{+}$. Anal. Calcd for $\mathrm{C}_{44} \mathrm{H}_{36} \mathrm{Br}_{4} \mathrm{~N}_{4} \mathrm{O}_{4}: \mathrm{C}, 52.62 ; \mathrm{H}$, 3.61; N, 5.58. Found. C, 52.65; H, 3.68; N, 5.55.

Ethyl 4-(3-methyl-5-styrylisoxazol-4-ylamino)-1,2,5,6tetrahydro-2,6-bis(2-hydroxyphenyl)-1-(3-methyl-5-styrylisoxazol-4-yl)pyridine-3-carboxylate (4h)

Brown solid; mp $203^{\circ} \mathrm{C}-205^{\circ} \mathrm{C}$. IR (KBr): 1657,3445 $\mathrm{cm}^{-1} .{ }^{1} \mathrm{H} \mathrm{NMR} \quad\left(300 \mathrm{MHz}, \mathrm{CDCl}_{3}\right): \delta=1.09(\mathrm{t}, 3 \mathrm{H}, \mathrm{J}=$ $\left.7.2 \mathrm{~Hz}, \mathrm{OCH}_{2} \mathrm{CH}_{3}\right), 2.30\left(\mathrm{~s}, 6 \mathrm{H}, 2\right.$ isoxazole- $\left.\mathrm{CH}_{3}\right), 2.41$ (dd, $1 \mathrm{H}, \mathrm{CH}, \mathrm{J}=15.2,2.4 \mathrm{~Hz}), 2.60(\mathrm{dd}, 1 \mathrm{H}, \mathrm{CH}, \mathrm{J}=$ $15.2,5.7 \mathrm{~Hz}), 4.11$ (q, $\left.2 \mathrm{H}, \mathrm{OCH}_{2} \mathrm{CH}_{3}\right), 5.18(\mathrm{~s}, 1 \mathrm{H}$, Ar-CH), 5.52 (t, $1 \mathrm{H}, \mathrm{Ar}-\mathrm{CH}), 6.62(\mathrm{~d}, 1 \mathrm{H}, \mathrm{CH}=\mathrm{CH}, \mathrm{J}=$ $12 \mathrm{~Hz}), 6.71(\mathrm{~d}, 1 \mathrm{H}, \mathrm{CH}=\mathrm{CH}, \mathrm{J}=12 \mathrm{~Hz}), 7.04-7.93$ (m, $18 \mathrm{H}, \mathrm{ArH}), 8.64$ (s, $1 \mathrm{H}, \mathrm{NH}, \mathrm{D}_{2} \mathrm{O}$ exchangeable), $9.10\left(\mathrm{~s}, 2 \mathrm{H}, 2 \mathrm{OH}, \mathrm{D}_{2} \mathrm{O}\right.$ exchangeable). ${ }^{13} \mathrm{C}$ NMR $(75$ MHz, DMSO-d $\left.\mathrm{d}_{6}\right): \delta=10.25,12.31,32.48,48.36,54.23$, $63.56,101.40,104.38,123.59$, 126.11, 127.52, 128.47, 129.36, 134.41, 134.69, 136.72, 139.24, 141.31, 151.40, 158.55. MS (ESI): $m / z=721.37[\mathrm{M}+\mathrm{H}]^{+}$. Anal. Calcd for $\mathrm{C}_{44} \mathrm{H}_{40} \mathrm{~N}_{4} \mathrm{O}_{6}$ : C, 73.32; H, 5.59; N, 7.77. Found. C, 73.37; $\mathrm{H}, 5.54 ; \mathrm{N}, 7.71$.

Ethyl 4-(3-methyl-5-styrylisoxazol-4-ylamino)-1,2,5,6tetrahydro-1-(3-methyl-5-styrylisoxazol-4-yl)-2,6-bis(2nitrophenyl)pyridine-3-carboxylate (4i)

Brown solid; mp $230^{\circ} \mathrm{C}-232^{\circ} \mathrm{C}$. IR (KBr): 1667,3448 $\mathrm{cm}^{-1} .{ }^{1} \mathrm{H}$ NMR $\left(300 \mathrm{MHz}, \mathrm{CDCl}_{3}\right): \delta=1.00(\mathrm{t}, 3 \mathrm{H}, \mathrm{J}=$ $\left.7.2 \mathrm{~Hz}, \mathrm{OCH}_{2} \mathrm{CH}_{3}\right), 2.30\left(\mathrm{~s}, 6 \mathrm{H}, 2\right.$ isoxazole- $\left.\mathrm{CH}_{3}\right), 2.40$ (dd, $1 \mathrm{H}, \mathrm{CH}, \mathrm{J}=15.2,2.4 \mathrm{~Hz}), 2.59$ (dd, $1 \mathrm{H}, \mathrm{CH}, \mathrm{J}=$ $15.2,5.7 \mathrm{~Hz}), 4.21$ (q, $\left.2 \mathrm{H}, \mathrm{OCH}_{2} \mathrm{CH}_{3}\right), 5.19$ (s, $1 \mathrm{H}$, Ar-CH), 5.63 (t, $1 \mathrm{H}, \mathrm{Ar}-\mathrm{CH}), 6.58(\mathrm{~d}, 1 \mathrm{H}, \mathrm{CH}=\mathrm{CH}, \mathrm{J}=$ $12 \mathrm{~Hz}), 6.61(\mathrm{~d}, 1 \mathrm{H}, \mathrm{CH}=\mathrm{CH}, \mathrm{J}=12 \mathrm{~Hz}), 7.00-7.85$ $(\mathrm{m}, 18 \mathrm{H}, \mathrm{ArH}), 8.85\left(\mathrm{~s}, 1 \mathrm{H}, \mathrm{NH}, \mathrm{D}_{2} \mathrm{O}\right.$ exchangeable). ${ }^{13} \mathrm{C}$ NMR (75 MHz, DMSO-d 6 ): $\delta=10.27,12.46$, $32.53,48.55,54.23,63.38,101.57,104.36,123.55$, $126.24,127.41,128.55,129.31,134.56,136.67,139.26$, $141.54,148.53,151.20,158.51$. MS (ESI): $m / z=781.26$ $[\mathrm{M}+\mathrm{H}]^{+}$. Anal. Calcd for $\mathrm{C}_{44} \mathrm{H}_{40} \mathrm{~N}_{6} \mathrm{O}_{8}: \mathrm{C}, 67.68 ; \mathrm{H}, 5.16$; N, 10.76. Found. C, 67.75; H, 5.19; N, 10.74.

Ethyl 4-(3-methyl-5-styrylisoxazol-4-ylamino)-1,2,5,6tetrahydro-1-(3-methyl-5-styrylisoxazol-4-yl)-2,6-bis(4nitrophenyl)pyridine-3-carboxylate $\mathbf{( 4 \mathbf { j } )}$

Pale brown solid; mp $237^{\circ} \mathrm{C}-239^{\circ} \mathrm{C}$. IR (KBr): 1655 ,
$3438 \mathrm{~cm}^{-1} .{ }^{1} \mathrm{H}$ NMR $\left(300 \mathrm{MHz}, \mathrm{CDCl}_{3}\right): \delta=1.05(\mathrm{t}, 3 \mathrm{H}$, $\left.\mathrm{J}=7.2 \mathrm{~Hz}, \mathrm{OCH}_{2} \mathrm{CH}_{3}\right), 2.26\left(\mathrm{~s}, 6 \mathrm{H}, 2\right.$ isoxazole- $\left.\mathrm{CH}_{3}\right)$, $2.42(\mathrm{dd}, 1 \mathrm{H}, \mathrm{CH}, \mathrm{J}=15.2,2.4 \mathrm{~Hz}), 2.51(\mathrm{dd}, 1 \mathrm{H}, \mathrm{CH}, \mathrm{J}$ $=15.2,5.7 \mathrm{~Hz}), 4.19\left(\mathrm{q}, 2 \mathrm{H}, \mathrm{OCH}_{2} \mathrm{CH}_{3}\right), 5.25(\mathrm{~s}, 1 \mathrm{H}$, Ar-CH), 5.59 (t, $1 \mathrm{H}, \mathrm{Ar}-\mathrm{CH}), 6.60$ (d, $1 \mathrm{H}, \mathrm{CH}=\mathrm{CH}, \mathrm{J}=$ $12 \mathrm{~Hz}), 6.73(\mathrm{~d}, 1 \mathrm{H}, \mathrm{CH}=\mathrm{CH}, \mathrm{J}=12 \mathrm{~Hz}), 6.97-7.85$ $(\mathrm{m}, 18 \mathrm{H}, \mathrm{ArH}), 8.51\left(\mathrm{~s}, 1 \mathrm{H}, \mathrm{NH}, \mathrm{D}_{2} \mathrm{O}\right.$ exchangeable). ${ }^{13} \mathrm{C}$ NMR $\left(75 \mathrm{MHz}, \mathrm{DMSO}-\mathrm{d}_{6}\right): \delta=10.22,12.41$, $32.58,48.63,54.25,63.32,101.48,104.40,123.57$, $126.26,128.11,128.71,129.32,134.54,136.62,139.26$, 141.56, 148.52, 151.49, 158.60. MS (ESI): $m / z=781.05$ $[\mathrm{M}+\mathrm{H}]^{+}$. Anal. Calcd for $\mathrm{C}_{44} \mathrm{H}_{40} \mathrm{~N}_{6} \mathrm{O}_{8}: \mathrm{C}, 67.68 ; \mathrm{H}, 5.16$; N, 10.76. Found. C, 67.70; H, 5.15; N, 10.77.

Ethyl 4-(3-methyl-5-styrylisoxazol-4-ylamino)-2,6-bis (4-(dimethylamino)phenyl)-1,2,5,6-tetrahydro-1-(3methyl-5-styrylisoxazol-4-yl)pyridine-3-carboxylate (4k)

Orange solid; $\mathrm{mp} 219^{\circ} \mathrm{C}-221^{\circ} \mathrm{C}$. IR (KBr): 1660 , $3441 \mathrm{~cm}^{-1}$. ${ }^{1} \mathrm{H}$ NMR $\left(300 \mathrm{MHz}, \mathrm{CDCl}_{3}\right): \delta=1.10(\mathrm{t}, 3 \mathrm{H}$, $\left.\mathrm{J}=7.2 \mathrm{~Hz}, \mathrm{OCH}_{2} \mathrm{CH}_{3}\right), 2.28\left(\mathrm{~s}, 6 \mathrm{H}, 2\right.$ isoxazole- $\left.\mathrm{CH}_{3}\right)$, $2.42(\mathrm{dd}, 1 \mathrm{H}, \mathrm{CH}, \mathrm{J}=15.2,2.4 \mathrm{~Hz}), 2.53(\mathrm{dd}, 1 \mathrm{H}, \mathrm{CH}, \mathrm{J}$ $=15.2,5.7 \mathrm{~Hz}), 3.85\left(\mathrm{~s}, 12 \mathrm{H}, 2 \mathrm{~N}\left(\mathrm{CH}_{3}\right)_{2}\right), 4.22(\mathrm{q}, 2 \mathrm{H}$, $\left.\mathrm{OCH}_{2} \mathrm{CH}_{3}\right), 5.27$ (s, $\left.1 \mathrm{H}, \mathrm{Ar}-\mathrm{CH}\right), 6.00$ (t, $\left.1 \mathrm{H}, \mathrm{Ar}-\mathrm{CH}\right)$, $6.63(\mathrm{~d}, 1 \mathrm{H}, \mathrm{CH}=\mathrm{CH}, \mathrm{J}=12 \mathrm{~Hz}), 6.71(\mathrm{~d}, 1 \mathrm{H}, \mathrm{CH}=$ $\mathrm{CH}, \mathrm{J}=12 \mathrm{~Hz}), 7.02-7.97(\mathrm{~m}, 18 \mathrm{H}, \mathrm{ArH}), 8.50$ (s, $1 \mathrm{H}$, $\mathrm{NH}, \mathrm{D}_{2} \mathrm{O}$ exchangeable). ${ }^{13} \mathrm{C}$ NMR $\left(75 \mathrm{MHz}, \mathrm{DMSO}-\mathrm{d}_{6}\right)$ : $\delta=10.23,12.43,32.37,40.55,48.51,54.23,63.38$, $101.40,104.32,123.41,126.26,128.18,128.54,129.36$, 134.58, 136.62, 139.21, 141.52, 149.71, 151.43, 158.67. MS (ESI): $m / z=775.11[\mathrm{M}+\mathrm{H}]^{+}$. Anal. Calcd for $\mathrm{C}_{48} \mathrm{H}_{50} \mathrm{~N}_{6} \mathrm{O}_{4}$ : C, 74.39; H, 6.50; N, 10.84. Found. C, 74.35; H, 6.51; N, 10.87 .

Ethyl 4-(3-methyl-5-styrylisoxazol-4-ylamino)-1,2,5,6tetrahydro-1-(3-methyl-5-styrylisoxazol-4-yl)-2,6-di(thiophen-2-yl)pyridine-3-carboxylate (4I)

Brown solid; $\mathrm{mp} 223^{\circ} \mathrm{C}-225^{\circ} \mathrm{C}$. IR (KBr): 1658,3436 $\mathrm{cm}^{-1}$. ${ }^{1} \mathrm{H}$ NMR $\left(300 \mathrm{MHz}, \mathrm{CDCl}_{3}\right): \delta=1.08(\mathrm{t}, 3 \mathrm{H}, \mathrm{J}=$ $\left.7.2 \mathrm{~Hz}, \mathrm{OCH}_{2} \mathrm{CH}_{3}\right), 2.20\left(\mathrm{~s}, 6 \mathrm{H}\right.$, 2isoxazole- $\left.\mathrm{CH}_{3}\right), 2.45$ (dd, $1 \mathrm{H}, \mathrm{CH}, \mathrm{J}=15.2,2.4 \mathrm{~Hz}), 2.59$ (dd, $1 \mathrm{H}, \mathrm{CH}, \mathrm{J}=$ 15.2, $5.7 \mathrm{~Hz}), 4.08$ (q, $\left.2 \mathrm{H}, \mathrm{OCH}_{2} \mathrm{CH}_{3}\right), 5.21(\mathrm{~s}, 1 \mathrm{H}$, Ar-CH), 6.10 (t, $1 \mathrm{H}, \mathrm{Ar}-\mathrm{CH}), 6.61(\mathrm{~d}, 1 \mathrm{H}, \mathrm{CH}=\mathrm{CH}, \mathrm{J}=$ $12 \mathrm{~Hz}), 6.73(\mathrm{~d}, 1 \mathrm{H}, \mathrm{CH}=\mathrm{CH}, \mathrm{J}=12 \mathrm{~Hz}), 6.81(\mathrm{dd}, 2 \mathrm{H}$, 2thiophene-H), 7.23 (d, $2 \mathrm{H}, 2$ thiophene-H), 7.31 (d, $2 \mathrm{H}$, 2thiophene-H), 7.52 - 7.97 (m, $10 \mathrm{H}, \mathrm{ArH}), 9.25(\mathrm{~s}, 1 \mathrm{H}$, $\mathrm{NH}, \mathrm{D}_{2} \mathrm{O}$ exchangeable). ${ }^{13} \mathrm{C}$ NMR $(75 \mathrm{MHz}$, DMSO-d 6 ): $\delta=10.12,12.22,32.39,48.40,54.16,63.55,101.51$, $104.23,123.18,123.50,126.22,126.86,127.10,129.31$, $134.57,136.43,140.12,141.61,151.24,158.50 . \mathrm{MS}$ (ESI): $m / z=701.33[\mathrm{M}+\mathrm{H}]^{+}$. Anal. Calcd for $\mathrm{C}_{40} \mathrm{H}_{36} \mathrm{~N}_{4} \mathrm{O}_{4} \mathrm{~S}_{2}: \mathrm{C}, 68.55 ; \mathrm{H}, 5.18 ; \mathrm{N}, 7.99$. Found. C, $68.57 ; \mathrm{H}, 5.13 ; \mathrm{N}, 7.96$.

Ethyl 4-(3-methyl-5-styrylisoxazol-4-ylamino)-1,2,5,6tetrahydro-1-(3-methyl-5-styrylisoxazol-4-yl)-2,6-di(1 Hpyrrol-2-yl)pyridine-3-carboxylate $(\mathbf{4 m})$

Brown solid; mp $227^{\circ} \mathrm{C}-229^{\circ} \mathrm{C}$. IR (KBr): 1660,3445 
$\mathrm{cm}^{-1} .{ }^{1} \mathrm{H}$ NMR $\left(300 \mathrm{MHz}, \mathrm{CDCl}_{3}\right): \delta=1.16(\mathrm{t}, 3 \mathrm{H}, \mathrm{J}=$ $7.2 \mathrm{~Hz}, \mathrm{OCH}_{2} \mathrm{CH}_{3}$ ), 2.30 (s, $6 \mathrm{H}, 2$ isoxazole- $\mathrm{CH}_{3}$ ), 2.48 $(\mathrm{dd}, 1 \mathrm{H}, \mathrm{CH}, \mathrm{J}=15.2,2.4 \mathrm{~Hz}), 2.61(\mathrm{dd}, 1 \mathrm{H}, \mathrm{CH}, \mathrm{J}=$ $15.2,5.7 \mathrm{~Hz}), 4.11\left(\mathrm{q}, 2 \mathrm{H}, \mathrm{OCH}_{2} \mathrm{CH}_{3}\right), 5.31(\mathrm{~s}, 1 \mathrm{H}$, Ar-CH), $6.20(\mathrm{t}, 1 \mathrm{H}, \mathrm{Ar}-\mathrm{CH}), 6.57(\mathrm{~d}, 1 \mathrm{H}, \mathrm{CH}=\mathrm{CH}, \mathrm{J}=$ $12 \mathrm{~Hz}), 6.68(\mathrm{~d}, 1 \mathrm{H}, \mathrm{CH}=\mathrm{CH}, \mathrm{J}=12 \mathrm{~Hz}), 6.73(\mathrm{dd}, 2 \mathrm{H}$, 2pyrrole-H), 7.21 (d, $2 \mathrm{H}, 2$ pyrrole-H), 7.30 (d, $2 \mathrm{H}$, 2pyrrole-H), $7.52-8.00$ (m, 10H, ArH), 8.72 (s, $2 \mathrm{H}$, 2pyrrole- $\mathrm{NH}, \mathrm{D}_{2} \mathrm{O}$ exchangeable), $9.00\left(\mathrm{~s}, 1 \mathrm{H}, \mathrm{NH}, \mathrm{D}_{2} \mathrm{O}\right.$ exchangeable). ${ }^{13} \mathrm{C}$ NMR $(75 \mathrm{MHz}$, DMSO-d 6 ): $\delta=10.33$, 12.30, 32.47, 48.50, 54.12, 63.42, 101.55, 104.32, 108.96, $109.11,118.86,123.57,126.24,129.32,131.12,134.58$, 136.50, 141.40, 151.43, 158.66. MS (ESI): $m / z=667.10$ $[\mathrm{M}+\mathrm{H}]^{+}$.Anal. Calcd for $\mathrm{C}_{40} \mathrm{H}_{38} \mathrm{~N}_{6} \mathrm{O}_{4}: \mathrm{C}, 72.05 ; \mathrm{H}, 5.74$; $\mathrm{N}, 12.60$. Found. C, 72.11; H, 5.71; N, 12.58 .

Ethyl 4-(3-methyl-5-styrylisoxazol-4-ylamino)-2,6-di (furan-2-yl)-1,2,5,6-tetrahydro-1-(3-methyl-5-styrylisoxazol-4-yl)pyridine-3-carboxylate (4n)

Pale yellow solid; $\mathrm{mp} 209^{\circ} \mathrm{C}-211^{\circ} \mathrm{C}$. IR $(\mathrm{KBr}): 1655$, $3432 \mathrm{~cm}^{-1} .{ }^{1} \mathrm{H}$ NMR $\left(300 \mathrm{MHz}, \mathrm{CDCl}_{3}\right): \delta=1.05(\mathrm{t}, 3 \mathrm{H}$, $\left.\mathrm{J}=7.2 \mathrm{~Hz}, \mathrm{OCH}_{2} \mathrm{CH}_{3}\right), 2.22\left(\mathrm{~s}, 6 \mathrm{H}, 2\right.$ isoxazole $\left.-\mathrm{CH}_{3}\right)$, $2.41(\mathrm{dd}, 1 \mathrm{H}, \mathrm{CH}, \mathrm{J}=15.2,2.4 \mathrm{~Hz}), 2.59$ (dd, $1 \mathrm{H}, \mathrm{CH}, \mathrm{J}$ $=15.2,5.7 \mathrm{~Hz}), 4.20\left(\mathrm{q}, 2 \mathrm{H}, \mathrm{OCH}_{2} \mathrm{CH}_{3}\right), 5.26(\mathrm{~s}, 1 \mathrm{H}$, Ar-CH), $6.18(\mathrm{t}, 1 \mathrm{H}, \mathrm{Ar}-\mathrm{CH}), 6.60(\mathrm{~d}, 1 \mathrm{H}, \mathrm{CH}=\mathrm{CH}, \mathrm{J}=$ $12 \mathrm{~Hz}), 6.74(\mathrm{~d}, 1 \mathrm{H}, \mathrm{CH}=\mathrm{CH}, \mathrm{J}=12 \mathrm{~Hz}), 6.69(\mathrm{dd}, 1 \mathrm{H}$, furan- $\mathrm{H}), 7.24(\mathrm{~d}, 1 \mathrm{H}$, furan- $\mathrm{H}), 7.42(\mathrm{~d}, 1 \mathrm{H}$, furan- $\mathrm{H})$, 7.50 - $7.9(\mathrm{~m}, 10 \mathrm{H}, \mathrm{ArH}), 8.92\left(\mathrm{~s}, 1 \mathrm{H}, \mathrm{NH}, \mathrm{D}_{2} \mathrm{O}\right.$ exchangeable). ${ }^{13} \mathrm{C}$ NMR (75 MHz, DMSO-d ${ }_{6}$ ): $\delta=10.26$, 12.22 , 32.34, 48.41, 54.10, 63.43, 101.41, 104.26, 106.77, $116.56,123.39,126.09,129.22,134.67,136.55,141.40$, $142.10,151.33,152.62,158.52$. MS (ESI): $\mathrm{m} / z=669.46$ $[\mathrm{M}+\mathrm{H}]^{+}$. Anal. Calcd for $\mathrm{C}_{40} \mathrm{H}_{36} \mathrm{~N}_{4} \mathrm{O}_{6}: \mathrm{C}, 71.84 ; \mathrm{H}, 5.43$; N, 8.38. Found. C, 71.88; H, 5.41; N, 8.32.

\section{REFERENCES}

[1] T. Welton, "Room-Temperature Ionic Liquids: Solvents for Synthesis and Catalysis," Chemical Reviews, 1999, p. 2701. doi:10.1021/cr1003248

[2] P. Wasserschid and W. Keim, "Ionic Liquids-New 'Solutions' for Transition Metal Catalysis," Angewandte Chemie International Edition, Vol. 39, No. 21, 2000, pp. 3772-3789. doi:10.1002/1521-3773(20001103)39:21<3772::AID-AN IE3772>3.0.CO;2-5

[3] R. Sheldon, "Catalytic Reactions in Ionic Liquids," Chemical Communications, No. 23, 2001, pp. 2399-2407. doi:10.1039/b107270f

[4] R. T. Coutts and A. F. Casay, "Pyridine and Its Derivatives: A Supplement," Inter Science Publishers, New York, 1975.

[5] M. Ferles and J. Pliml, "Adavances in Heterocyclic Chemistry,” Academic Press, New York, 1970.

[6] J. W. Langston, P. Ballard, J. W. Tetrud and I. Irwin, "Chronic Parkinsonism in Humans Due to a Product of
Meperidine-Analog Synthesis," Science, Vol. 219, No. 4587, 1983, pp. 979-980.

doi:10.1126/science.6823561

[7] J. W. Langston, "MPTP Neurotoxicity: An Overview and Characterization of Phases of Toxicity," Life Science, Vol. 36, No. 3, 1985, pp. 201-206.

doi:10.1016/0024-3205(85)90059-1

[8] C. C. Chiueh, R. S. Burns, S. P. Markey, D. M. Jacobowitz and I. J. Kopin, "A Primate Model of Parkinsonism: Selective Destruction of Dopaminergic Neurons in the Pars Compacta of the Substantia Nigra by N-Methyl-4phenyl-1,2,3,6-tetrahydropyridine," Life Science, Vol. 36, 1985 , pp. 213-217.

[9] J. W. Langston, L. S. Forno, C. S. Rebert and I. Irwin, "Selective Nigral Toxicity after Systemic Administration of 1-Methyl-4-phenyl-1,2,5,6-Tetrahydropyrine (MPTP) in the Squirrel Monkey," British Research, Vol. 292, 1984, pp. 390-394.

[10] J. E. Christie, A. Shering, J. Ferguson and A. I. M. Glen, "Physostigmine and Arecoline: Effects of Intravenous Infusions in Alzheimer Presenile Dementia," The British Journal of Psychiatry, Vol. 138, 1981, pp. 46-50. doi:10.1192/bjp.138.1.46

[11] P. Sauerberg, J. J. Larsen, E. Falch and P. KrogsgaardLarsen, "A Novel Class of Conformationally Restricted Heterocyclic Muscarinic Agonists," Journal of Medicinal Chemistry, Vol. 29, No. 6, 1986, pp. 1004-1009. doi: $10.1021 / \mathrm{jm} 00156 \mathrm{a} 018$

[12] P. Sauerberg, B. Fjalland, J. J. Larsen, T. Bach-Lauritsen, E. Falch and P. Krogsgaard-Larsen, "Pharmacological Profile of a Novel Class of Muscarinic Acetylcholine Receptor Agonists," European Journal of Pharmacology, Vol. 130, No. 1-2, 1986, pp. 125-131. doi:10.1016/0014-2999(86)90191-3

[13] P. Krogsgaard-Larsen, E. Falch, P. Sauerberg, S. B. Freedman, H. L. Lembùl and E. Meier, "In Subtypes of Muscarinic Receptors III," Elsevier, Amsterdam, 1988, pp. 69-74.

[14] P. Sauerberg, E. Falch, E. Meier, H. L. Lembùl and P. Krogsgaard-Larsen, "Heterocyclic Muscarinic Agonists. Synthesis and Biological Activity of Some Bicyclic Sulfonium Arecoline Bioisosteres," Journal of Medicinal Chemistry, Vol. 31, No. 7, 1988, pp. 1312-1316. doi: $10.1021 / \mathrm{jm} 00402 \mathrm{a} 010$

[15] T. Ogamino and S. Nishiyama, "A New Ring-Opening Access to Aeroplysinin-1, a Secondary Metabolite of Verongia Aerophoba," Tetrahedron, Vol. 59, 2003, pp. 94199423.

[16] G. Daidone, D. Raffa, B. Maggio, F. Plescia, V. M. C. Cutuli, N. G. Mangano and A. Caruso, "Synthesis and Antiproliferative Activity of Novel 3-(Indazol-3-yl)-quinazolin-4(3H)-one and 3-(Indazol-3-yl)-benzotriazin-4(3H)one Derivatives," Arch. Pharm. Med. Chem, Vol. 50, 1999, p. 332.

[17] K. Tomita, Y. Takahi, R. Ishizuka, S. Kamamura, M. Nakagawa, M. Ando, T. Nakanishi, T. Nakamura and H. Udaira, Ann. Sankyo Res. Lab. Vol. 1, 1973, p. 25; Chem. Abstr. 1974, 80, 120808.

[18] J. J. Talley, D. L. Brown, J. S. Carter, M. J. Graneto, C. 
M. Koboldt, J. L. Masferrer, W. E. Perkins, R. S. Rogers, A. F. Shaffer, Y. Y. Zhang, B. S. Zweifel and K. Seibert, "4-[5-Methyl-3-phenylisoxazol-4-yl]-benzenesulfonamid e, Valdecoxib: A Potent and Selective Inhibitor of COX-2," Journal of Medicinal Chemistry, Vol. 43, 2000, pp. 775-777.

[19] K. Haripara, S. Patel, A. Joshi and H. Parekh, "Synthesis and Biological Evaluation of Some Cyano Pyridines and Isoxazoles," Indian J. Het. Chem, Vol. 13, 2004, pp. 221224.

[20] H. Uno, M. Kurokawa, Y. Masuda and H. Nishimura, "Studies on 3-Substituted 1,2-Benzisoxazole Derivatives. Syntheses of 3-(Sulfamoylmethyl)-1,2-Benzisoxazole Derivatives and Their Anticonvulsant Activities," Journal of Medicinal Chemistry, Vol. 22, No. 2, 1979, pp. 180-183. doi:10.1021/jm00188a011

[21] W.-T. Li, D.-R. Hwang, C.-P. Chen, C.-W. Shen, C.-L. Huang, T.-W. Chen, C.-H. Lin, Y.-L. Chang, Y.-Y. Chang, Y.-K. Lo, H.-Y. Tseng, C.-C. Lin, J.-S. Song, H.-C. Chen, S.-J. Chen, S.-H. Wu and C.-T. Chen, "Synthesis and Biological Evaluation of N-Heterocyclic Indolyl Glyoxylamides as Orally Active Anticancer Agents," Journal of Medicinal Chemistry, Vol. 46, No. 9, 2003, pp. 1706-1715. doi:10.1021/jm020471r

[22] E. Rajanarendar, M. Nagi Reddy, K. Rama Murthy, K. Govardhan Reddy, S. Raju, M. Srinivas, B. Praveen and M. Srinivasa Rao, "Synthesis, Antimicrobial, and Mosquito Larvicidal Activity of 1-Aryl-4-Methyl-3,6-Bis(5-Methylisoxazol-3-yl)-2-Thioxo-2,3,6,10b-Tetrahydro1H-Pyrimido[5,4-c]quinolin-5-Ones," Bioorganic \& Medicinal Chemistry Letters, Vol. 20, No. 20, 2010, pp. 6052-6055. doi:10.1016/j.bmcl.2010.08.060

[23] E. Rajanarendar, M. Nagi Reddy, S. Rama Krishna, K. Rama Murthy, P. Surendar, R. N. Reddy and Y. N. Reddy, "Synthesis and in Vitro and in Vivo Anticancer Activity of Novel Phenylmethylene Bis-isoxazolo[4,5-b]azepines," Bioorganic \& Medicinal Chemistry Letters, Vol. 22, No. 2012, pp. 149-153. doi:10.1016/j.bmcl.2011.11.044

[24] E. Rajanarendar, S. Raju, M. Nagi Reddy, S. Rama Krishna, L. Hari Kiran, A. Ram Narasimha Reddy and Y Narasimha Reddy, "Multi-Component Synthesis and in Vitro and in Vivo Anticancer Activity of Novel Aryl- methylene Bis-isoxazolo[4,5-b]pyridine- $N$-Oxides," European Journal of Medicinal Chemistry, Vol. 50, 2012, pp. 274-279.

[25] E. Rajanarendar, M. Nagi Reddy, S. Rama Krishna, K. Govardhan Reddy, Y. N. Reddy and M. V. Rajam, "Design, Synthesis, in Vitro Antimicrobial and Anticancer Activity of Novel Methylenebis-Isoxazolo[4,5-b]azepines Derivatives," European Journal of Medicinal Chemistry, Vol. 50, 2012, pp. 344-349. doi:10.1016/j.ejmech.2012.02.013

[26] E. Rajanarendar, M. Nagi Reddy, K. Govardhan Reddy and S. Rama Krishna, "l-Proline Catalyzed Efficient OnePot Three-Component Aza-Diels-Alder Reactions on Nitrostyrylisoxazoles: A Facile Synthesis of New Isoxazolyl Tetrahydroquinolines and Isoxazolo[2,3-a]pyrimidines," Tetrahedron Letters, Vol. 53, No. 23, 2012, pp. 29092913. doi:10.1016/j.tetlet.2012.04.002

[27] M. Misra, S. K. Pandey, V. P. Pandey, J. Pandey, R. Tri- pathi, R. P. Tripathi, "Organocatalyzed Highly Atom Economic One Pot Synthesis of Tetrahydropyridines as Antimalarials," Bioorganic \& Medicinal Chemistry, Vol. 17, No. 2, 2009, pp. 625-633. doi:10.1016/j.bmc.2008.11.062

[28] A. T. Khan, T. Parvin and L. H. Choudhary, "Effects of Substituents in the $\beta$-Position of 1,3-Dicarbonyl Compounds in Bromodimethylsulfonium Bromide-Catalyzed Multicomponent Reactions: A Facile Access to Functionalized Piperidines," The Journal of Organic Chemistry, Vol. 73, No. 1, 2008, pp. 8398-8402. doi:10.1021/jo8014962

[29] A. T. Khan, M. Lal and M. M. Khan, "Synthesis of Highly Functionalized Piperidines by One-Pot Multicomponent Reaction Using Tetrabutylammonium Tribromide (TBATB)," Tetrahedron Letters, Vol. 51, No. 33, 2010, pp. 4419-4424. doi:10.1016/j.tetlet.2010.06.069

[30] A. T. Khan, M. M. Khan and K. R. B. Karthi, "Iodine Catalyzed One-Pot Five-Component Reactions for Direct Synthesis of Densely Functionalized Piperidines," Tetrahedron, Vol. 66, No. 39, 2010, pp. 7762-7772. doi:10.1016/j.tet.2010.07.075

[31] P. A. Clarke, P. A. Zaytzev and A. C. Whitwood, "Pot, Atom and Step Economic (PASE) Synthesis of Highly Functionalized Piperidines: A Five-Component Condensation," Tetrahedron Letters, Vol. 48, No. 20, 2007, pp. 5209-5212. doi:10.1016/j.tetlet.2007.05.141

[32] S. Mishra, R. Ghosh, "Efficient One-Pot Synthesis of Functionalized Piperidine Scaffolds via $\mathrm{ZrOCl}_{2} \cdot 8 \mathrm{H}_{2} \mathrm{O}$ Catalyzed Tandem Reactions of Aromatic Aldehydes with Amines and Acetoacetic Esters," Tetrahedron Letters, Vol. 52, No. 22, 2011, pp. 2857-2861. doi:10.1016/j.tetlet.2011.03.116

[33] W. Hong-Juan, M. Li-Ping and Z. Zhan-Hui, ACS Comb. Sci. Vol. 13, 2011, p. 181.

[34] R. Kumar, P. Chaudhary, S. Nimesh, A. K. Verma and R. Chandra, "An Efficient Synthesis of 1,5-Benzadiazepine Derivatives Catalyzed by Silver Nitrate," Green Chemistry, Vol. 8, No. 6, 2006, pp. 519-521. doi:10.1039/b601993e

[35] A. K. Verma, P. Attri, V. Chopra, R. K. Tiwari and R. Chandra, "Triethylammonium Acetate (TEAA): A Recyclable Inexpensive Ionic Liquid Promotes the Chemoselective Aza- and Thia-Michael Reactions," Monatsh Chemistry, Vol. 139, 2008, p. 1041

[36] C. Wang, L. Guo, H. Li, Y. Wang, J. Weng and L. Wu, "Preparation of Simple Ammonium Ionic Liquids and Their Application in the Cracking of Dialkoxypropanes," Green Chemistry, Vol. 8, No. 7, 2006, pp. 603-607. doi: $10.1039 / \mathrm{b} 600041 \mathrm{j}$

[37] J. Weng, C. Wang, H. Li, Y. Wang, "Novel Quaternary Ammonium Ionic Liquids and Their Use as Dual SolventCatalysts in the Hydrolytic Reaction," Green Chemistry, Vol. 8, No. 1, 2006, pp. 96-99. doi:10.1039/b508325g

[38] H. Jiang, C. Wang, H. Li and Y. Wang, "Preparation of Dialkoxypropanes in Simple Ammonium Ionic Liquids," Green Chemistry, Vol. 8, No. 12, 2006, pp. 1076-1079. doi:10.1039/b608249a 